BMJ Open Sport \& Exercise Medicine

\title{
Effects of almond, dried grape and dried cranberry consumption on endurance exercise performance, recovery and psychomotor speed: protocol of a randomised controlled trial
}

\author{
Noah M A d'Unienville, ${ }^{01}$ Alison M Hill, ${ }^{2}$ Alison M Coates, ${ }^{1}$ Catherine Yandell, ${ }^{1}$ \\ Maximillian J Nelson, ${ }^{1}$ Jonathan D Buckley ${ }^{1}$
}

To cite: d'Unienville NMA Hill AM, Coates AM, et al. Effects of almond, dried grape and dried cranberry consumption on endurance exercise performance, recovery and psychomotor speed: protocol of a randomised controlled trial. BMJ Open Sport \& Exercise Medicine 2019;5:e000560. doi:10.1136/ bmjsem-2019-000560

Accepted 11 July 2019

\section{Check for updates}

(c) Author(s) (or their employer(s)) 2019. Re-use permitted under CC BY-NC. No commercial re-use. See rights and permissions. Published by BMJ.

${ }^{1}$ School of Health Science, University of South Australia, Adelaide, South Australia, Australia

${ }^{2}$ School of Pharmacy and Medical Sciences, University of South Australia, Adelaide, South Australia, Australia

Correspondence to Noah M A d'Unienville; noah.dunienville@mymail.unisa. edu.au

\begin{abstract}
Background Foods rich in nutrients, such as nitrate, nitrite, L-arginine and polyphenols, can promote the synthesis of nitric oxide (NO), which may induce ergogenic effects on endurance exercise performance. Thus, consuming foods rich in these components, such as almonds, dried grapes and dried cranberries (AGC), may improve athletic performance. Additionally, the antioxidant properties of these foods may reduce oxidative damage induced by intense exercise, thus improving recovery and reducing fatigue from strenuous physical training. Improvements in NO synthesis may also promote cerebral blood flow, which may improve cognitive function.
\end{abstract}

Methods and analysis Ninety-six trained male cyclists or triathletes will be randomised to consume $\sim 2550 \mathrm{~kJ}$ of either a mixture of AGC or a comparator snack food (oat bar) for 4 weeks during an overreaching endurance training protocol comprised of a 2-week heavy training phase, followed by a 2-week taper. The primary outcome is endurance exercise performance (5 min time-trial performance) and secondary outcomes include markers of NO synthesis (plasma and urinary nitrites and nitrates), muscle damage (serum creatine kinase and lactate dehydrogenase), oxidative stress (F2-isoprostanes), endurance exercise function (exercise efficiency, submaximal oxygen consumption and substrate utilisation), markers of internal training load (subjective well-being, rating of perceived exertion, maximal rate of heart rate increase and peak heart rate) and psychomotor speed (choice reaction time).

Conclusion This study will evaluate whether consuming AGC improves endurance exercise performance, recovery and psychomotor speed across an endurance training programme, and evaluate the mechanisms responsible for any improvement.

Trial registration number ACTRN12618000360213.

\section{INTRODUCTION}

A key determinant of endurance exercise performance is the ability of active muscle to use oxygen. Strategies that increase oxygen delivery to, and the utilisation of oxygen in the mitochondria may thus improve endurance performance. Nitric oxide (NO) mediates skeletal muscle contractile properties, ${ }^{1}$ endothelial function, and mitochondrial biogenesis and respiration, ${ }^{2}$ and therefore, increasing NO synthesis may improve muscle blood flow and oxygen delivery and utilisation during exercise (figure 1). ${ }^{34}$ These changes may lead to enhanced exercise efficiency, reduced oxygen deficit ${ }^{56}$ and enhanced exercise tolerance, ${ }^{7}$ all of which may improve endurance performance.

Exercise performance may also be improved by enhancing postexercise recovery, which may enable greater tolerance to training. One approach to improving training tolerance is to increase the availability of antioxidants which neutralise exercise-induced free radicals and thereby have the potential to reduce muscle damage. ${ }^{9}$ Additionally, NO may promote postexercise muscle repair through activation of satellite cells involved in muscle remodelling and hypertrophy, ${ }^{10}$ and through increased blood flow, which may enhance nutrient replenishment and muscle protein synthesis in fatigued muscle. ${ }^{11}$

Factors which impact on fatigue, recovery and exercise performance may also affect cardiovascular and cognitive function. Exercise-induced muscle damage may affect cardiovascular control by stimulating group III/IV afferent neurons, which relay intramuscular conditions to supraspinal cardiovascular centres. ${ }^{1213}$ Through this afferent signalling, muscle damage has been proposed as a key mediator of changes in the maximal rate of heart rate increase (rHRI), a novel marker of fatigue status. ${ }^{14-20}$ Several studies have demonstrated that rHRI is slowed when exercise performance is reduced, and faster when performance is improved, but no direct 
L-arginine (almonds) [26]
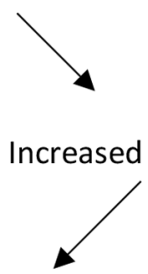

Improved blood flow

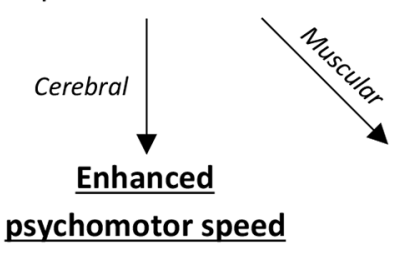

$\Delta$

Dietary nitrite (cranberries) [38]

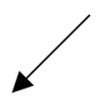

Via increased endothelial nitric

oxide synthase activity [19]

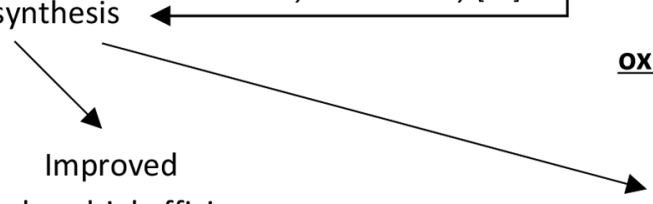

mitochondrial efficiency

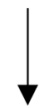

Enhanced endurance performance

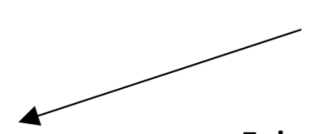

Enhanced recovery (reduced fatigue)
Polyphenols, vitamins E \& C, \& antioxidant minerals (almonds, sultanas $\&$ cranberries)

[27-32,35-37]

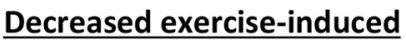

oxidative stress (decreased F2-isoprostanes)

(decreased lactate dehydrogenase and creatine kinase)

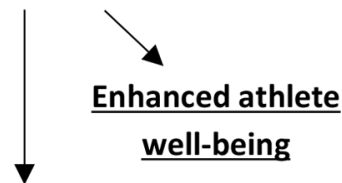

Reduced slowing of rHR

Figure 1 Proposed effects of AGC consumption. AGC, almonds, dried grapes and dried cranberries; rHRI, maximal rate of heart rate increase.

evidence exists for mechanisms behind such changes. NO might also increase cerebrovascular dilatation and blood flow, ${ }^{21}{ }^{22}$ which may promote psychomotor speed (figure 1), which is important for decision-making and movement execution, and is critical for many athletic pursuits. Further, as exercise-induced fatigue can slow psychomotor speed, ${ }^{23} 24$ improving recovery by elevating NO availability might promote maintenance of psychomotor speed.

$\mathrm{NO}$ is synthesised from nitrite $\left(\mathrm{NO}_{2}^{-}\right)$, nitrate $\left(\mathrm{NO}_{3}^{-}\right.$ ), or L-arginine in a reaction catalysed by NO synthase (NOS). NOS activity can be increased through the dietary intake of polyphenols. ${ }^{25}$ Thus, consuming foods that are a source of L-arginine, $\mathrm{NO}_{2}^{-}, \mathrm{NO}_{3}^{-}$or polyphenols may increase NO synthesis, which has potential to induce improvements in mitochondrial efficiency and muscle recovery, while foods containing antioxidants may attenuate muscle damage induced by free radicals. Almonds are a rich source of arginine, ${ }^{26}$ polyphenols ${ }^{2728}$ and antioxidants. ${ }^{27-29}$ Grapes are also a rich source of polyphenols and antioxidants. ${ }^{30-32}$ Preliminary evidence suggests that consumption of almonds ${ }^{33}$ and grape juice ${ }^{34}$ improves endurance performance. Cranberries are another source of polyphenols, antioxidants and $\mathrm{NO}_{2}^{-}$ ${ }^{35-38}$ but limited studies have investigated their effects on exercise performance. ${ }^{39}$ The proposed physiological effects of consuming almonds, dried grapes and dried cranberries (AGC) are illustrated in figure 1.

The aim of this study is to evaluate whether a combination of AGC, which are good sources of polyphenols, antioxidants, L-arginine and $\mathrm{NO}_{2}^{-}$, reduces exercise-induced oxidative damage to contracting muscle, promotes exercise performance and recovery, speeds rHRI and enhances psychomotor speed during exercise.

\section{METHODS AND ANALYSIS}

This study will be a 5-week, single-blind, randomised, controlled, parallel-arm trial evaluating the effects of consuming a mixture of almonds, dried grapes (sultanas) and dried cranberries (AGC) compared with an isocaloric comparator (food) on endurance exercise performance, psychomotor speed, rHRI and markers of NO availability, oxidative stress and muscle damage in trained male cyclists or triathletes.

Participants will first attend a familiarisation session where they will perform an incremental exercise test to determine their maximum power output $\left(\mathrm{W}_{\max }\right)$ and be familiarised with all aspects of the study. Training intensities for the subsequent training periods will be prescribed based on percentages of maximum HR achieved during the $\mathrm{W}_{\max }$ test. Participants will then undertake a 1-week run-in period of light training (LT), before baseline assessments of NO availability, oxidative stress, muscle damage, endurance exercise performance, rHRI, psychomotor speed and well-being are performed. They will then perform 2 weeks of heavy training (HT) followed by a 2-week taper $(\mathrm{T})$, with assessments repeated post-HT and post-T.

After baseline testing, participants will be randomly allocated to consume either an AGC mix or an alternative isocaloric snack food (oat bar) daily for 4 weeks during HT and T. Each food will provide $2550 \mathrm{~kJ} /$ day of energy.

An overview of the study design is displayed in figure 2.

\section{Primary research aims}

1. To investigate the effects of AGC consumption on endurance exercise performance.

2. To investigate the effects of AGC consumption on recovery, as assessed by changes in exercise performance following an increase in training load. 
Daily consumption of $\sim 2550 \mathrm{~kJ}$ of AGC or comparator food

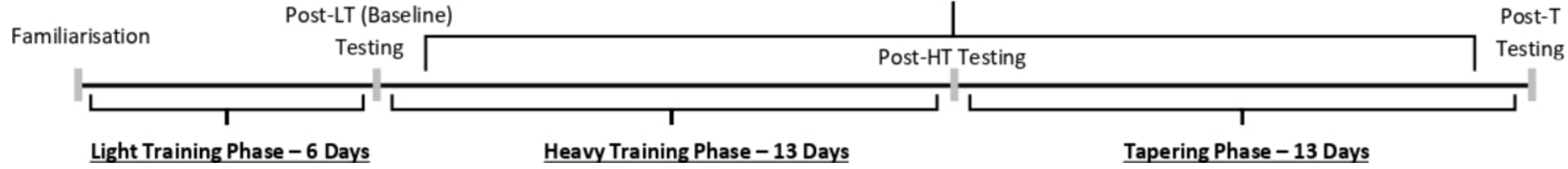

Figure 2 Study overview. AGC, almonds, dried grapes and dried cranberries; HT, heavy training; LT, light training; T, taper training.

\section{Secondary research aims}

1. To investigate the effects of AGC on physiological correlates of endurance performance.

a. To determine whether AGC consumption can increase levels of urinary and plasma nitrates and nitrites, and whether these changes are correlated with changes in endurance performance.

2. To evaluate whether changes in markers of oxidative and muscle damage (F2-isoprostanes, and creatine kinase $(\mathrm{CK})$ and lactate dehydrogenase $(\mathrm{LDH})$ ) are associated with improved endurance performance, recovery and well-being.

3. To investigate the effects of AGC consumption on psychomotor speed (choice reaction time) at rest and during exercise.

4. To evaluate whether changes in rHRI are correlated with changes in muscle damage markers.

\section{Recruitment and screening}

Participants will be in recruited in Adelaide, Australia, via advertisements sent to local cycling and triathlon club social media pages, posted on the University of South Australia social media pages, Facebook and posted at local cafes and cycling races. Participants who complete all aspects of the study will receive an honorarium of \$AUD350 to compensate for their time and travel expenses. Participants who do not complete the study will be reimbursed on a pro-rata basis. Those who express interest will be sent an information sheet and Diet and Lifestyle Questionnaire (DLQ) requesting information regarding general health status, medication and/or supplement use, cycling training history and diet. Responses from the DLQ will then be assessed to evaluate participant eligibility.

\section{Inclusion criteria}

Participants will be eligible for the study if they meet all the following criteria:

1. Male aged $18-60$ years.

2. Trained cyclist or triathlete ( $\geq 6$ months training and $\geq$ three times per week).

3. Qualify as low risk according to the Exercise and Sport Science Australia, Sports Medicine Australia and Fitness Australia Adult Pre-Exercise Health Screening Tool (2011).

4. During the 4 weeks prior to participation, they have not consumed following:

a. $>30 \mathrm{~g} /$ day of nuts or nut products (eg, butter, meal, oil, etc). b. $>50 \mathrm{~g} /$ day of grapes and/or sultanas or $>1 \mathrm{~L} /$ week of grape juice.

c. $>50 \mathrm{~g} /$ day fresh or dried cranberries or $>1 \mathrm{~L} /$ week of cranberry juice.

5. No allergies to any of the study foods.

\section{Exclusion criteria}

Participants will be excluded from the study if they meet any of the following criteria:

1. Have regularly smoked during the last 6 months.

2. Have a gastrointestinal disorder that affects nutrient absorption.

3. Regularly use supplements or medications that may impact study outcomes (eg, beetroot juice, anti-inflammatory medications, drugs affecting HR, etc).

4. Show unwillingness to be randomised to either experimental group.

5. Fail to satisfy the investigator regarding suitability to participate for any other reason.

6. Are unwilling or unable to provide written consent.

\section{Treatment allocation and blinding}

A toss of a coin will determine which dietary group the first participant is randomised to. Following this, participants will be allocated to treatment by minimisation ${ }^{40}$ based on 5 min time-trial (5TT) performance (in kJ/ $\mathrm{kg}$ ), body mass index and age at familiarisation. Minimisation will ensure balanced characteristics between the treatment groups at baseline, and has been proposed as the best randomisation method for smaller clinical trials, such as the proposed study. ${ }^{40}{ }^{41}$ As the participants will consume whole foods that are easily identifiable, they cannot be blinded. However, researchers conducting assessments and statistical analyses will be blinded (ie, single-blind) to prevent bias.

\section{Familiarisation}

Written informed consent will be obtained from participants prior to participation.

At the familiarisation visit only, participants will perform an incremental cycling exercise test to exhaustion on an electronically braked cycle ergometer (Lode Excalibur Sport, Lode BV, Groningen, the Netherlands) to determine $\mathrm{W}_{\max }$. The $\mathrm{W}_{\max }$ test will be preceded by a 5 min warm-up at $120 \mathrm{~W}$ (to allow for determination of rHRI), after which power output will be increased every 2.5 min until exhaustion. ${ }^{42} \mathrm{~W}_{\max }$ will then be determined according to the following formula: 


$$
\mathrm{W}_{\text {max }}=\mathrm{W}_{\text {out }}+(\mathrm{t} / 150) * 25
$$

where $\mathrm{W}_{\text {out }}$ is the workload of the last completed stage and $t$ is the time in seconds in the final stage.

$\mathrm{W}_{\max }$ assessment will be used to prescribe exercise intensity for all subsequent sustained effort tests (SETs), while maximum $\mathrm{HR}$ attained during $\mathrm{W}_{\max }$ assessment will be used to prescribe training intensities based on set percentages of maximum HR. Participants will then be familiarised with all other aspects of the study protocol, including consuming a small portion of both the test and comparator foods to ensure the foods are palatable to them, and that they do not suffer any allergic reaction.

\section{Dietary intervention}

Participants will be instructed to consume AGC or comparator food as a snack throughout the day, but otherwise not change their background diet during the study. Participants will be provided with a 2 week supply of AGC or comparator food at baseline (ie, post-LT) and post-HT testing points. Participants in both treatment groups will be asked to avoid consumption of nuts, grapes and cranberries, or food/drink products containing these during the intervention period, other than the experimental foods provided to them if they are allocated to the intervention group.

Participants in the intervention group will consume $75 \mathrm{~g} /$ day of raw, natural, unsalted almonds, $25 \mathrm{~g} /$ day of dried grapes (sultanas) and $25 \mathrm{~g}$ /day of dried cranberries, providing an energy intake of $2550 \mathrm{~kJ}$. Participants will be provided with preweighed daily portions of each component of the AGC to ensure consistency in quantities consumed. Four weeks of supplementation with this quantity of almonds has previously been shown to improve endurance performance in elite cyclists. ${ }^{33}$ Additionally, grape juice has been shown to improve endurance exercise performance,${ }^{34}$ and while previous studies have not investigated the potential of dried grapes or cranberries for improving endurance performance, it is hypothesised that their polyphenol, antioxidant and $\mathrm{NO}_{2}^{-30-32} 35-38$ content will facilitate performance adaptations.

Participants in the comparator group will consume $132 \mathrm{~g}$ (3.3 bars) per day of oat bars (Golden Oat Baked Oaty Slices, Mother Earth, Hamilton, New Zealand), which will also provide $2550 \mathrm{~kJ}$. Thus, the comparator is isocaloric to AGC but does not contain the proposed ergogenic nutrients (ie, arginine, polyphenols, $\mathrm{NO}_{2}$ and antioxidants). The nutritional profile of both the AGC and comparator treatments is presented in table 1. While the phytochemical and antioxidant properties of AGC have been reported previously, samples of almonds, sultanas and dried cranberries consumed in the proposed study will be assessed for total phytochemical content and antioxidant activities according to techniques previously described ${ }^{43}{ }^{44}$ Total phenolic content will be determined using the Folin-Ciocalteu's phenol reagent, whereas the total flavonoid content will be assessed using aluminium chloride solution. For antioxidant activities,
Table 1 Treatment group nutritional information

AGC mix (75 g

raw, unsalted

almonds, $25 \mathrm{~g}$

dried cranberries (132 $\mathrm{g}$ Mother

and $25 \mathrm{~g}$

dried grapes

(sultanas))

Earth Golden

Oat Baked

Oaty Slices)

\begin{tabular}{lrl}
\hline Energy (kJ) & 2550.1 & 2550.0 \\
\hline Protein (g) & 16.5 & 9.9 \\
\hline Total fat (g) & 42.3 & 32.4 \\
Saturated fat (g) & 2.9 & 20.6 \\
\hline Polyunsaturated fat (g) & 10.0 & $\mathrm{~N} / \mathrm{A}$ \\
\hline Monounsaturated fat (g) & 27.4 & $\mathrm{~N} / \mathrm{A}$ \\
\hline Carbohydrates (g) & 39.9 & 65.4 \\
Sugars (g) & 37.5 & 29.7 \\
\hline Dietary fibre (g) & 8.1 & 9.9 \\
\hline Arginine (mg) & $1848.8^{*}$ & N/A \\
\hline Total polyphenols (mg) & $560.3 \dagger$ & N/A \\
Flavonoids (mg) & $14.84 \ddagger$ & N/A \\
\hline
\end{tabular}

Unless specified otherwise, data are sourced from the Foodworks Nutritional Analysis Software V.9 (Xyris Software, Pty Ltd., Brisbane, Australia).

*US Department of Agriculture, Agricultural Research Service, Nutrient Data Laboratory. USDA National Nutrient Database for Standard Reference, Legacy. Version Current: April 2018. †Rothwell JA, Pérez-Jiménez J, Neveu V, Medina-Ramon A, M'Hiri N, Garcia Lobato P, Manach C, Knox K, Eisner R, Wishart D, Scalbert A. 2013. Phenol-Explorer 3.0: a major update of the Phenol-Explorer database to incorporate data on the effects of food processing on polyphenol content. Database, 10.1093/ database/bat070.

łHaytowitz DB, Wu X, Bhagwat S. 2018. USDA Database for the Flavonoid Content of Selected Foods, Release 3.3. U.S. Department of Agriculture, Agricultural Research Service. Version Current: March 2018.

$\mathrm{N} / \mathrm{A}$, not available.

1,1-diphenyl-2-picrylhydrazyl antioxidant activity and ferric-reducing antioxidant power will be determined as previously described.$^{43}{ }^{44}$ The $2,2^{\prime}$-azinobis (3-ethylbenzothiazoline-6-sulfonic acid) and oxygen radical absorbance capacity will be assessed based on modified protocols of Re $e t a t^{45}$ and Gillespie $e t a t^{46}$, respectively.

\section{Cycling training intervention}

To induce training adaptations that will improve endurance exercise performance (ie, 5TT performance), participants will perform a standardised, three-phase endurance training programme. This will begin with a 6-day run-in phase of LT designed to provide stable baseline measures. Participants will then complete a 13-day HT phase that is designed to induce substantial fatigue (ie, a state of overreaching ${ }^{47}$ ), followed by a 13-day T phase that will provide sufficient recovery to allow for supercompensatory adaptations in response to the HT, with a resultant increase in endurance exercise performance. 
Table 2 Study training prescription

\begin{tabular}{|c|c|c|}
\hline Light training (LT) & Heavy training $(\mathrm{HT})$ & Taper training (T) \\
\hline $\begin{array}{l}\text { Day 1, } 3 \text { and } 5: 65 \%-75 \% \text { max HR } \\
\text { (60 min) }\end{array}$ & $\begin{array}{l}\text { Every day: } \\
\text { Warm-up: <69\% max HR (10 min) }\end{array}$ & $\begin{array}{l}\text { Day } 1,7 \text { and } 13: \text { Rest } \\
\text { Day 2, 4, 6, 8, } 9 \text { and 12: 65\%-75\% max HR } \\
\text { (60 min) }\end{array}$ \\
\hline $\begin{array}{l}\text { Day } 2 \text { and } 4: 75 \%-85 \% \text { max HR } \\
\text { (30 min) }\end{array}$ & $\begin{array}{l}\text { Zone } 2: 69 \%-81 \% \max \mathrm{HR}(8.5 \mathrm{~min}) \\
\text { Zone 3: } 82 \%-87 \% \max \mathrm{HR}(8 \mathrm{~min}) \\
\text { Zone 4: } 88 \%-94 \% \max \mathrm{HR}(7.5 \mathrm{~min}) \\
\text { Zone 5: >94\% max HR (3min) }\end{array}$ & $\begin{array}{l}\text { Day 3: 65\%-75\% max HR ( } 40 \text { min) } \\
\text { Day } 5 \text { \& 10: } 75 \%-85 \% \text { max HR (50 mins) }\end{array}$ \\
\hline
\end{tabular}

max HR, maximum heart rate; mins, minutes.

This protocol has previously been used to induce fatigue and supercompensation in several previous studies run in our laboratory. ${ }^{15-20}$ The comparison of changes in outcome measures from post-LT to post-HT between the AGC and comparator groups will determine the effect of AGC on minimising fatigue (ie, improving recovery), while the comparison of post-LT to post-T measures will determine their effect on improving endurance exercise performance.

The training prescriptions for LT, HT and $\mathrm{T}$ are provided in table 2, with prescribed intensities for training sessions based on percentages of maximum HR achieved during the $\mathrm{W}_{\text {max }}$ test at familiarisation (detailed below). To ensure compliance with the training prescription, participants will record HR during each training session using a personal HR monitor (RS800CX, Polar Electro Oy, Kempele, Finland). Participants will also record a rating of perceived exertion (RPE) for each training session. The overall training load will be determined by the summation of each session's training impulse (TRIMP) from recorded HR data. ${ }^{48}$ All training will be performed on participants' own bicycles attached to a stationary bicycle trainer (JetBlack Whisper Drive Plus, JetBlack, Rouse Hill, Australia).

\section{Training and diet record}

Participants will record their daily consumption of test foods and the duration and sessional RPE (Borg 6-20 RPE scale ${ }^{49}$ ) of their training sessions to calculate compliance with test food consumption and exercise prescription. They will also be given a list of foods high in polyphenols, $\mathrm{NO}_{3}^{-}$and $\mathrm{NO}_{2}^{-3850-52}$ and asked to record their daily intakes of these foods to determine if existing and/or additional dietary intake of polyphenols, $\mathrm{NO}_{3}^{-}$ and $\mathrm{NO}_{2}^{-}$influences the ergogenic and/or physiological effects of AGC.

\section{Dietary and training compliance}

Participants will be required to consume at least $70 \%$ of each component of the AGC. If unable to consume their daily requirements of AGC on any given day, they will be allowed to increase their intake over the following day(s) to compensate. Using the completed training and diet record, percentage compliance with test food consumption will be calculated from 'total grams consumed' and 'total number of grams prescribed'. Training compliance will be calculated in Microsoft Excel using 'completed TRIMP' and 'prescribed minimum TRIMP'. Participants are required to attain at least $70 \%$ of the prescribed TRIMP during LT and T. Full adherence to the very high loads prescribed for HT may not be possible for some athletes due to fatigue and/or fatigue-induced changes in cardiac autonomic regulation that can impair HR increases and thus make achieving high target HRs difficult. Thus, for HT, compliance will be based on RPE, with participants having to report an RPE of at least 15 for each session for them to be deemed to be compliant with the training protocol. Participants will not be made aware of this latter requirement to avoid training bias.

\section{Testing protocol}

An illustration of the testing protocol performed following LT, HT and T is displayed in figure 3.

\section{Pretesting restrictions}

During the 24 hours prior to testing visits, participants will be required to abstain from alcohol and any moderate or vigorous physical activity not prescribed by their training. They must also fast overnight and refrain from caffeine consumption in the 12 hours prior to testing.

\section{Biochemical analyses}

Fasting blood samples will be obtained via venepuncture from the median cubital vein, while urinary measures will be determined from a 24 hours urine collection. Plasma samples will be prepared in accordance to Barden et $a \tilde{l}^{\tilde{3}}$ and, along with urine samples, will be analysed for F2-isoprostanes as a marker of oxidative stress, as well as $\mathrm{NO}_{2}^{-}$and $\mathrm{NO}_{3}^{-}$levels. ${ }^{545}$ Serum samples will be analysed for $\mathrm{LDH}$ and $\mathrm{CK}$, as markers of muscle damage using an automated analyser with commercial kits (Konelab 20XTi, Thermo Fisher Scientific, Waltham, Massachusetts, USA).

Daily analysis of life demands for athletes questionnaire

To assess the well-being of participants, they will complete a modified daily analysis of life demands for athletes 


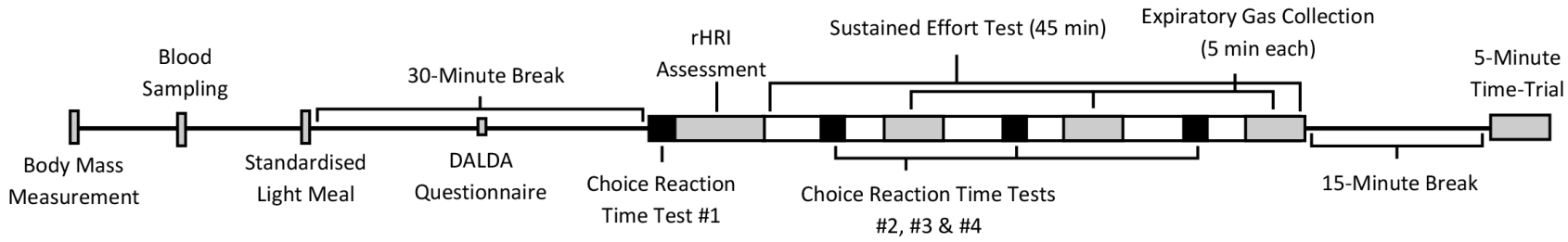

Figure 3 Overview of the testing protocol performed. DALDA, daily analysis of life demands for athletes; rHRI, maximal rate of heart rate increase.

(DALDA) questionnaire, which will provide subjective measures of various components of their psychological and physiological status. ${ }^{56}$ Responses to Part A and B of the DALDA require 'normal', 'better than normal' or 'worse than normal' responses and will be used to determine an overall DALDA score, while responses to Part C (scaled responses) will provide separate outcome measures of mood, energy, soreness, fatigue and stress.

\section{Psychomotor speed}

Prior to performing any exercise, and at the 5, 20 and 35 min marks of the Sustained Exertion Test (see below), participants will perform the Deary-Liewald reaction time task to assess choice reaction time as a measure of psychomotor speed. ${ }^{2324}$ Conducting this test while participants are exercising and in various states of fatigue throughout the study is designed to better emulate decision-making in an applied sport setting compared with performing the test at rest. Data will be expressed as number of errors, the mean response time and SD of all correct response times.

\section{rHRI assessment}

rHRI will be assessed during the 5 min warm-up preceding the SET. Participants will rest on the cycle ergometer for 4-6 min to stabilise HR, before being instructed to begin cycling at $120 \mathrm{~W}$ for $5 \mathrm{~min}$, while $\mathrm{HR}$ is recorded as R-R intervals. Participants will not be warned of when they will begin cycling to prevent an anticipatory increase in HR prior to exercise ${ }^{57} \mathrm{rHRI}$ will then be calculated using $30 \mathrm{~s}$ of HR data prior to commencing exercise and $3 \mathrm{~min}$ of exercise data according to the method of Bellenger $e t$ $a .^{20}$

\section{Sustained exertion test}

On completion of rHRI assessment, participants will cycle at $70 \% \mathrm{~W}_{\max }$ for $45 \mathrm{~min}$. Five minutes of expiratory gases will be collected after 10,25 and $40 \mathrm{~min}$ using the TrueOne 2400 (Parvo Medics, Utah, USA) for determination of volume of oxygen consumption $\left(\mathrm{VO}_{2}\right)$ as a measure of exercise economy. Respiratory exchange ratio will be recorded to represent substrate use, and gross exercise efficiency will be determined by dividing work output by energy expenditure, with energy expenditure calculated from the tables of Zuntz and Schumberg. ${ }^{58}$

\section{Five-minute time-trial}

Following a $15 \mathrm{~min}$ recovery period after completing the SET, participants will perform a 5TT on the cycle ergometer. This will involve performing as much work as possible in $5 \mathrm{~min}$. The total work performed per unit body mass $(\mathrm{kJ} / \mathrm{kg})$ during the $5 \mathrm{TT}$ will represent the measure of exercise performance. Peak $\mathrm{VO}_{2}$ (recorded during $15 \mathrm{~s}$ intervals) and peak HR attained during the 5TT will also be recorded. The 5TT has excellent reliability with a $1.2 \%$ coefficient of variation. ${ }^{16}$

\section{Statistical analysis}

\section{Sample size}

In two recent studies conducted in our laboratory which evaluated the effects of a similar HT period followed by a $\mathrm{T}$ on 5TT performance, the average work done at the end of the T was $107.7 \mathrm{~kJ}$ with a pooled SD of $9.84 \mathrm{~kJ} .{ }^{17} 18$ Yi et $_{a} \mathrm{l}^{33}$ reported a $5.3 \%$ greater TT performance (effect size of 0.58 ) compared with placebo following 4 weeks of consuming $75 \mathrm{~g}$ /day of almonds. Thus, demonstrating a similar magnitude of increase in 5TT performance in the present study as being statistically significant in a two-tailed test with $80 \%$ power and at an $\alpha$-level of 0.05 would require 96 participants to complete the study (48 AGC mix and 48 comparator foods). A total of 108 participants will be recruited to account for a drop out of $\sim 10 \%$. This is a conservative power analysis given that, in addition to using the same dose of almonds used by Yi et $a l l^{33}$ in the present study participants will also consume dried grapes and dried cranberries, which may potentially induce a greater effect on performance.

\section{Data analysis}

Data will be presented as mean \pm SD for descriptive statistics and as means $\pm \mathrm{SE}$ for reporting estimated effects. Statistical analysis will be performed using Stata/IC V.15.1 (StataCorp LLC, College Station, Texas, USA). The effects of AGC and comparator foods on the dependent measures over time will be analysed using linear mixed effects models, with fixed effects entered as outcome measures, treatment allocation and time point, and participant ID entered as a random effect. All covariances in the covariance matrix will be set to zero. Covariates will include age, training compliance and dietary intervention compliance. If participants drop out, their available data will be used in the analysis provided the data due to drop out are missing at random. The random effects mixed model will use all remaining available data in the 
analysis, and this will constitute an intention-to-treat analysis. A sensitivity analysis will then be performed using only data from participants who completed all aspects of the protocol (ie, no missing data). If data are normally distributed, relationships will be assessed using Pearson's correlation coefficient. If data are not normally distributed, it will be log-transformed and analysed using non-parametric analyses. Repeated measures analyses will be used to evaluate relationships between different parameters. Statistical significance will be set at an alpha level of 0.05 .

\section{Patient and public involvement}

There was no patient or public involvement in the design of this study.

\section{DISCUSSION}

While the preliminary work of Yi $e t a l^{33}$ demonstrated the potential of almond consumption for improving endurance exercise performance, there remains a need for further investigation of their efficacy, including whether this may be influenced by age and fitness level. In this study, the shorter duration of the 5TT should assist with identifying any ergogenic effects of AGC consumption, ${ }^{59}$ as the positive effects of NO-related supplements for performance appear most evident during shorter rather than longer-duration performance tests. ${ }^{59} 60$ However, improvements in oxygen economy and/or exercise efficiency may infer the potential of AGC to enhance endurance performance over longer durations.

Significant associations between the improvements in exercise performance and increases in plasma and urinary nitrate and/or nitrite levels, without concomitant improvements in oxidative stress and muscle damage, would suggest that any increases in endurance performance may be caused by enhancement in muscular blood flow and metabolic function, rather than reductions in oxidative stress and muscle damage. Alternatively, inverse associations between oxidative stress or muscle damage and endurance performance would suggest that any ergogenic effects of AGC consumption may be due to effects on attenuating muscle damage. In addition to the beneficial effects that reductions in oxidative stress and muscle damage may have on physical performance, it is also possible that these effects would result in benefits for athlete well-being.

Measures of muscle damage will also be valuable in elucidating potential mechanisms underlying the relationship between rHRI and endurance performance. Recent evidence suggests that rHRI may be slowed when performance is impaired as a result of signals originating from the fatigued muscle that are transmitted to the cardiovascular centre in the brain stem via group III and IV afferent neurons, ${ }^{14}$ which modulate cardiac acceleration at exercise onset. ${ }^{12}{ }^{13}$ However, this hypothesis is presently primarily based on speculation, and evaluating the effects of muscle damage on rHRI will provide evidence that will assist in either supporting or refuting this hypothesis. Furthermore, associations between rHRI and endurance within the proposed study will continue to provide evidence regarding the efficacy of using rHRI to monitor fatigue in endurance athletes.

Finally, despite positive findings on the benefits of nut and berry consumption on cognitive decline in older adults, ${ }^{61-64}$ their potential for improving cognitive performance in younger, athletic populations, and under conditions of fatigue that are more relevant to athletes, remains unknown. In addition to evaluating the effect of AGC on psychomotor speed during conditions of acute fatigue (as participants progress through the SET), the study will also investigate whether AGC can attenuate impairments in psychomotor speed that may be induced by periods of fatigue-inducing intense training. ${ }^{23} 24$

This protocol paper describes the methodology that will be used to evaluate the effects of AGC consumption on components of endurance exercise performance, recovery and psychomotor speed following periods of heavy and tapered training (ie, an overreaching cycle). In addition, the study will evaluate whether any effects on these outcomes are mediated by NO, oxidative stress and/or muscle damage. Most previous studies investigating the effects of foods on endurance performance have not been structured across an overreaching cycle. The potential to determine whether a dietary intervention (using AGC) can reduce training-induced fatigue (ie, improve recovery) and enhance peak performance is a unique element of this study. Thus, the findings of the present study may have numerous implications for athletes.

Acknowledgements The Almond Board of California donated the almonds used in the study and Mother Earth donated the oat bars.

Contributors JB initiated the study. JB, AC, AH, MN, $C Y$ and NMAd' $U$ designed the study. JB, AC and AH secured the funding. NMAd'U prepared the manuscript, which was reviewed by all the authors.

Funding This work was supported by a grant from the International Nut and Dried Fruit Council Foundation. NMAd'U is supported by a Research Training Program (Domestic) Scholarship from the Australian Department of Education and Training.

Competing interests This study is funded by a grant from the International Nut and Dried Fruit Council Foundation, although they have not and will not be involved in the collection, analysis and interpretation of data, or the preparation or submission of the article for publication. JB invented the rHRI technology that will be used in this study. The rHRI technology has been patented by the University of South Australia and JB has assigned all the rights of this technology to the University. AC has previously provided consultancy services to Nuts For Life, an Australian initiative established to provide information about the health effects of tree nuts.

Patient consent for publication Not required.

Ethics and dissemination The study has been approved by the University of South Australia Human Research Ethics Committee and registered with the Australia and New Zealand Clinical Trials Registry.

Provenance and peer review Not commissioned; externally peer reviewed. Data availability statement There are no data in this work.

Open access This is an open access article distributed in accordance with the Creative Commons Attribution Non Commercial (CC BY-NC 4.0) license, which permits others to distribute, remix, adapt, build upon this work non-commercially, and license their derivative works on different terms, provided the original work is properly cited, appropriate credit is given, any changes made indicated, and the use is non-commercial. See: http://creativecommons.org/licenses/by-nc/4.0/. 


\section{REFERENCES}

1. Coggan AR, Peterson LR. Dietary nitrate enhances the contractile properties of human skeletal muscle. Exerc Sport Sci Rev 2018;46:254-61.

2. Stamler JS, Meissner G. Physiology of nitric oxide in skeletal muscle. Physiol Rev 2001;81:209-37.

3. Bescós R, Sureda A, Tur JA, et al. The effect of nitric-oxide-related supplements on human performance. Sports Med 2012;42:99-117.

4. Lara J, Ashor AW, Oggioni C, et al. Effects of inorganic nitrate and beetroot supplementation on endothelial function: a systematic review and meta-analysis. Eur J Nutr 2016;55:451-9.

5. Bailey SJ, Varnham RL, DiMenna FJ, et al. Inorganic nitrate supplementation improves muscle oxygenation, $\mathrm{O}_{2}$ uptake kinetics, and exercise tolerance at high but not low pedal rates. J Appl Physiol 2015;118:1396-405.

6. Breese BC, McNarry MA, Marwood S, et al. Beetroot juice supplementation speeds $\mathrm{O}_{2}$ uptake kinetics and improves exercise tolerance during severe-intensity exercise initiated from an elevated metabolic rate. Am J Physiol Regul Integr Comp Physiol 2013;305:R1441-R1450.

7. Jones AM. Dietary nitrate supplementation and exercise performance. Sports Med 2014;44:35-45.

8. Buonocore D, Negro M, Arcelli E, et al. Anti-Inflammatory dietary interventions and supplements to improve performance during athletic training. J Am Coll Nutr 2015;34 Suppl 1:62-7.

9. Bentley DJ, Ackerman J, Clifford T, et al. Acute and Chronic Effects of Antioxidant Supplementation on Exercise Performance.. In: Lamprecht M, ed. Antioxidantsin sport nutrition. Boca Raton, FL: CRC Press/ Taylor \& Francis (c) 2015 by Taylor \& Francis Group, LLC, 2015.

10. Radak Z, Zhao Z, Koltai E, et al. Oxygen consumption and usage during physical exercise: the balance between oxidative stress and ROS-dependent adaptive signaling. Antioxid Redox Signal 2013:18:1208-46.

11. Deane CS, Wilkinson DJ, Phillips BE, et al. "Nutraceuticals" in relation to human skeletal muscle and exercise. Am J Physiol Endocrinol Metab 2017;312:E282-E299.

12. Amann M, Blain GM, Proctor LT, et al. Group III and IV muscle afferents contribute to ventilatory and cardiovascular response to rhythmic exercise in humans. J Appl Physiol 2010;109:966-76.

13. Nobrega ACL, O'Leary D, Silva BM, et al. Neural regulation of cardiovascular response to exercise: role of central command and peripheral afferents. Biomed Res Int 2014;2014:1-20.

14. Nelson MJ, Bellenger CR, Thomson RL. Slowing of cardiac acceleration following acute fatigue is predominantly peripherally mediated, exercise and sport science Australia research to practice 2016. Melbourne: Melbourne Convention \& Exhibition Centre, 2016: 74. p. 74

15. Nelson MJ, Bellenger CR, Thomson RL, et al. Maximal rate of heart rate increase correlates with fatigue/recovery status in female cyclists. Eur J Appl Physiol 2017;117:2425-31.

16. Nelson MJ, Thomson RL, Rogers DK, et al. Maximal rate of increase in heart rate during the rest-exercise transition tracks reductions in exercise performance when training load is increased. J Sci Med Sport 2014;17:129-33.

17. Bellenger $\mathrm{CR}$, Thomson RL, Howe PRC, et al. Monitoring athletic training status using the maximal rate of heart rate increase. J Sci Med Sport 2016;19:590-5.

18. Bellenger $\mathrm{C}$, Thomson RL, Robertson EY, et al. The effect of functional overreaching on parameters of autonomic heart rate regulation. Eur J Appl Physiol 2017:1-10.

19. Bellenger $C R$, Karavirta L, Thomson RL, et al. Contextualizing parasympathetic hyperactivity in functionally Overreached athletes with perceptions of training tolerance. Int J Sports Physiol Perform 2016;11:685-92.

20. Bellenger CR, Thomson RL, Davison K, et al. Optimization of maximal rate of heart rate increase assessment in runners. Res $Q$ Exerc Sport 2018;89:322-31.

21. Thompson C, Wylie LJ, Fulford J, et al. Dietary nitrate improves sprint performance and cognitive function during prolonged intermittent exercise. Eur J Appl Physiol 2015;115:1825-34.

22. Wightman EL, Haskell-Ramsay CF, Thompson KG, et al. Dietary nitrate modulates cerebral blood flow parameters and cognitive performance in humans: a double-blind, placebo-controlled, crossover investigation. Physiol Behav 2015;149:149-58.

23. Rietjens GJWM, Kuipers H, Adam JJ, et al. Physiological, biochemical and psychological markers of strenuous traininginduced fatigue. Int J Sports Med 2005;26:16-26.

24. Nederhof E, Lemmink K, Zwerver J, et al. The effect of high load training on psychomotor speed. Int J Sports Med 2007;28:595-601.

25. Kim J, Auger C, Schini-Kerth V. Activation of eNOS by polyphenolrich products and polyphenolic compounds. Curr Pharm Des 2014;20:3521-9.
26. Ahrens S, Venkatachalam M, Mistry AM, et al. Almond (Prunus dulcis L.) protein quality. Plant Foods Hum Nutr 2005;60:123-8.

27. Chen C-YO, Blumberg JB. Phytochemical composition of nuts. Asia Pac J Clin Nutr 2008:17(Suppl 1):329-32.

28. Milbury PE, Chen C-Y, Dolnikowski GG, et al. Determination of flavonoids and phenolics and their distribution in almonds. J Agric Food Chem 2006;54:5027-33.

29. Yada S, Lapsley K, Huang G. A review of composition studies of cultivated almonds: macronutrients and micronutrients. J Food Compos Anal 2011;24:469-80.

30. Parker TL, Wang X-H, Pazmiño J, et al. Antioxidant capacity and phenolic content of grapes, sun-dried raisins, and golden raisins and their effect on ex vivo serum antioxidant capacity. J Agric Food Chem 2007;55:8472-7.

31. Kelebek H, Jourdes M, Selli S, et al. Comparative evaluation of the phenolic content and antioxidant capacity of sun-dried raisins. J Sci Food Agric 2013:93:2963-72.

32. Williamson G, Carughi A. Polyphenol content and health benefits of raisins. Nutr Res 2010;30:511-9.

33. Yi M, Fu J, Zhou L, et al. The effect of almond consumption on elements of endurance exercise performance in trained athletes. $J$ Int Soc Sports Nutr 2014;11:18.

34. Toscano LT, Tavares RL, Toscano LT, et al. Potential ergogenic activity of grape juice in runners. Appl Physiol Nutr Metab 2015;40:899-906.

35. Blumberg JB, Camesano TA, Cassidy A, et al. Cranberries and their bioactive constituents in human health. Adv Nutr 2013;4:618-32.

36. Marais J, Khoo C. Polyphenolic content of sweet dried cranberries compared to cranberry juice cocktail. FASEB J 2013;27(1 Suppl):19-79.

37. Côté J, Caillet S, Doyon G, et al. Bioactive compounds in cranberries and their biological properties. Crit Rev Food Sci Nutr 2010;50:666-79

38. Hord NG, Tang Y, Bryan NS. Food sources of nitrates and nitrites: the physiologic context for potential health benefits. Am J Clin Nutr 2009;90:1-10.

39. Skarpańska - Stejnborn A, Basta P, Trzeciak J, et al. Effects of cranberry (Vaccinum macrocarpon) supplementation on iron status and inflammatory markers in rowers. J Int Soc Sports Nutr 2017;14:7.

40. Altman DG, Bland JM. Treatment allocation by minimisation. BM $2005 ; 330$

41. Taves DR. The use of minimization in clinical trials. Contemp Clin Trials 2010;31:180-4.

42. Jeukendrup A, Saris WIMHM, Brouns F, et al. A new validated endurance performance test. Med Sci Sports Exerc 1996;28:266-70.

43. Deo P, Hewawasam E, Karakoulakis A, et al. In vitro inhibitory activities of selected Australian medicinal plant extracts against protein glycation, angiotensin converting enzyme (ACE) and digestive enzymes linked to type II diabetes. BMC Complement Altern Med 2016;16:435.

44. Koch ER, Deo P. Nutritional supplements modulate fluorescent protein-bound advanced glycation endproducts and digestive enzymes related to type 2 diabetes mellitus. BMC Complement Altern Med 2016;16:338

45. Re R, Pellegrini N, Proteggente A, et al. Antioxidant activity applying an improved ABTS radical cation decolorization assay. Free Radic Biol Med 1999;26:1231-7.

46. Gillespie KM, Chae JM, Ainsworth EA. Rapid measurement of total antioxidant capacity in plants. Nat Protoc 2007;2:867-70.

47. Halson SL, Jeukendrup AE. Does Overtraining exist? Sports Med 2004:34:967-81.

48. Banister E. Modeling elite athletic performance. In: MacDougal $\mathrm{J}$, Wenger $\mathrm{H}$, Green $\mathrm{H}$, eds. Physiological testing of the high performance athlete. Champaign, IL: HumanKinetics, 1991.

49. Borg G. Perceived exertion as an indicator of somatic stress. Scand J Rehabil Med 1970;2:92-8.

50. Blekkenhorst LC, Prince RL, Ward NC, et al. Development of a reference database for assessing dietary nitrate in vegetables. development of a reference database for assessing dietary nitrate in vegetables. Mol Nutr Food Res 2017;61:1600982.

51. Pérez-Jiménez J, Neveu V, Vos F, et al. Identification of the 100 richest dietary sources of polyphenols: an application of the Phenol-Explorer database. Eur J Clin Nutr 2010;64 Suppl 3:S112-S120.

52. Floegel A, Kim D-O, Chung S-J, et al. Comparison of ABTS/DPPH assays to measure antioxidant capacity in popular antioxidant-rich us foods. J Food Compos Anal 2011;24:1043-8.

53. Barden AE, Mas E, Croft KD, et al. Minimizing artifactual elevation of lipid peroxidation products (F2-isoprostanes) in plasma during collection and storage. Anal Biochem 2014;449:129-31.

54. Mori TA, Croft KD, Puddey IB, et al. An improved method for the measurement of urinary and plasma F2-isoprostanes 
using gas Chromatography-Mass spectrometry. Anal Biochem 1999;268:117-25.

55. Yang $X$, Bondonno CP, Indrawan A, et al. An improved mass spectrometry-based measurement of $\mathrm{NO}$ metabolites in biological fluids. Free Radic Biol Med 2013;56:1-8.

56. Rushall BS. A tool for measuring stress tolerance in elite athletes. $J$ Appl Sport Psychol 1990;2:51-66.

57. Stuttering APA. Emotions, and heart rate during anticipatory anxiety: a critical review. J Fluency Disord 2004;29:123-33.

58. ZuntzN, Schumburg W. Studien zu einer Physiologie des Marsches. Berlin: August Hirschwald, 1901.

59. Shannon OM, Barlow MJ, Duckworth L, et al. Dietary nitrate supplementation enhances short but not longer duration running time-trial performance. Eur J Appl Physiol 2017;117:775-85.
60. Domínguez R, Maté-Muñoz JL, Cuenca E, et al. Effects of beetroot juice supplementation on intermittent high-intensity exercise efforts. $J$ Int Soc Sports Nutr 2018;15:2.

61. Cherniack EP. A berry thought-provoking idea: the potential role of plant polyphenols in the treatment of age-related cognitive disorders. Br J Nutr 2012;108:794-800.

62. Vauzour D, Rodriguez-Mateos A, Corona G, et al. Polyphenols and human health: prevention of disease and mechanisms of action. Nutrients 2010;2:1106-31.

63. Valls-Pedret C, Lamuela-Raventós RM, Medina-Remón A, et al Polyphenol-rich foods in the Mediterranean diet are associated with better cognitive function in elderly subjects at high cardiovascular risk. J Alzheimers Dis 2012:29:773-82.

64. Valls-Pedret C, Sala-Vila A, Serra-Mir M, et al. Mediterranean diet and age-related cognitive decline: a randomized clinical trial. JAMA Intern Med 2015;175:1094-103. 
Correction: Effects of almond, dried grape and dried cranberry consumption on endurance exercise performance, recovery and psychomotor speed: protocol of a randomised controlled trial

d'Unienville NMA, Hill AM, Coates AM, et al. Effects of almond, dried grape and dried cranberry consumption on endurance exercise performance, recovery and psychomotor speed: protocol of a randomised controlled trial. BMJ Open Sp Ex Med 2019;5:e000560. doi: 10.1136/bmjsem-2019-000560.

The published version misspelled the authors' names. The correct names should be as follows.

Noah M A d'Unienville, Alison M Hill, Alison M Coates, Catherine Yandell, Maximillian J Nelson, Jonathan D Buckley

Open access This is an open access article distributed in accordance with the Creative Commons Attribution Non Commercial (CC BY-NC 4.0) license, which permits others to distribute, remix, adapt, build upon this work non-commercially, and license their derivative works on different terms, provided the original work is properly cited, appropriate credit is given, any changes made indicated, and the use is non-commercial. See: http://creativecommons.org/licenses/by-nc/4.0/.

@ A Author(s) (or their employer(s)) 2019. Re-use permitted under CC BY-NC. No commercial re-use. See rights and permissions. Published by BMJ.

BMJ Open Sp Ex Med 2019;5:e000560corr1. doi:10.1136/bmjsem-2019-000560corr1

Check for updates 